\title{
Relative O- and X-mode transmitted power from SuperDARN as it relates to the RRI instrument on ePOP
}

\author{
R. G. Gillies ${ }^{1}$, G. C. Hussey ${ }^{1}$, G. J. Sofko ${ }^{1}$, and H. G. James ${ }^{2}$ \\ ${ }^{1}$ Institute for Space and Atmospheric Studies, University of Saskatchewan, Saskatoon, Saskatchewan, Canada \\ ${ }^{2}$ Communication Research Centre Canada, Ottawa, Ontario, Canada
}

Received: 27 October 2009 - Revised: 15 March 2010 - Accepted: 16 March 2010 - Published: 24 March 2010

\begin{abstract}
The Cascade Demonstrator Small-Sat and Ionospheric Polar Explorer (CASSIOPE) satellite is scheduled to be launched in 2010. On board this satellite will be a suite of eight scientific instruments comprising the enhanced Polar Outflow Probe (ePOP). One instrument is the Radio Receiver Instrument (RRI) which will be used to receive HF transmissions from various ground transmitters such as the Super Dual Auroral Radar Network (SuperDARN) array. Magnetoionic polarization and propagation theory have been used to model the relative power that SuperDARN delivers to the Ordinary $(\mathrm{O})$ and Extraordinary $(\mathrm{X})$ modes of propagation. These calculations have been performed for various frequencies in the SuperDARN transmitting band and for all five Canadian based SuperDARN radars. The geometry of the radars with respect to the background magnetic field results in the $\mathrm{X}$-mode dominating the transmitted signal when the modelled wave propagates northward and is nearly perpendicular to the magnetic field lines. Other propagation directions (i.e., above or southwards of the radar) results in propagation which is anti-parallel to the magnetic field lines and an equal splitting of transmitted power between the $\mathrm{O}$ - and $\mathrm{X}$-modes occurs. The modelling analysis shows that for either high transmitting frequencies or low ionospheric electron densities, the range of latitudes that signal will be received is quite large (up to $\sim 90^{\circ}$ of latitude). Also for these conditions, the range of elevations where the $\mathrm{X}$-mode signal strongly dominates the O-mode signal will be apparent in the received signal. Conversely, for lower transmitting frequencies or higher ionospheric electron densities, the latitudinal range that signal will be received over is smaller. Here the $\mathrm{X}$-mode-only band is not apparent in the received signal as both modes will be received with roughly equal power. These relative mode power calculations can be used to characterize
\end{abstract}

Correspondence to: R. G. Gillies (rob.gillies@usask.ca) the average electron density content in the ionosphere or provide a measure of relative absorption in the $\mathrm{D}$ - and E-regions when the satellite passes through the field-of-view of a SuperDARN radar.

Keywords. Radio science (Radio wave propagation; Instruments and techniques)

\section{Introduction}

The Canadian designed and built Cascade Demonstrator Small-Sat and Ionospheric Polar Explorer (CASSIOPE) small satellite will be launched in 2010 (Yau et al., 2006). The satellite bus will consist of two payloads, a commercial data storage and forward system called Cascade and a scientific group of instruments called the enhanced Polar Outflow Probe (ePOP). ePOP will consist of various particle detectors, active and passive radio experiments, an auroral imager, a magnetic field detector, and a GPS system. The scientific goals of ePOP are to study outflows of particles from the polar regions, radio wave propagation, and ionospheric tomography (Yau et al., 2006). Some radio experiments will use the Radio Receiver Instrument (RRI) on ePOP (James, 2003, 2006; James and Lunscher, 2006; James et al., 2006). The RRI is a radio receiver that is fed by four 3-m dipole antennas. Each antenna can operate as a separate monopole, or in pairs as two crossed (perpendicular) 6-m dipoles. The RRI will measure electric fields between $10 \mathrm{~Hz}$ and $18 \mathrm{MHz}$. One RRI experiment to be undertaken will include various ground-based HF transmitters such as the Super Dual Auroral Radar Network (SuperDARN) radars (Greenwald et al., 1995), which will transmit HF waves to be detected by the RRI as ePOP orbits overhead.

One objective of the SuperDARN-RRI experiment is the detection of various ionospheric structures through interpretation of the signal received by the RRI. The background

Published by Copernicus Publications on behalf of the European Geosciences Union. 


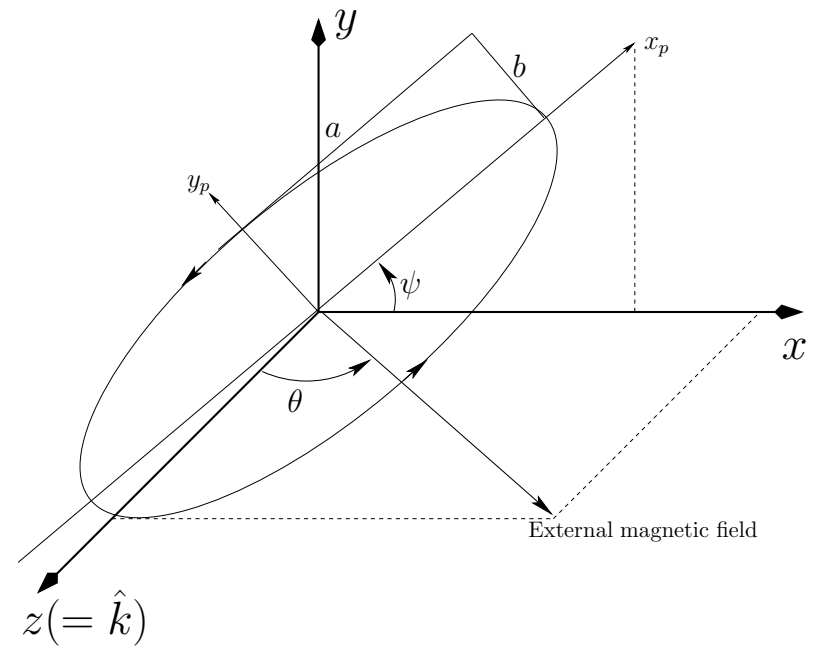

Fig. 1. The coordinate system and a general polarization ellipse. The orientation angle is represented by $\psi, a$ represents the semimajor axis, $b$ represents the semi-minor axis, and $\theta$ is the aspect angle. The propagation direction of the wave $\hat{\boldsymbol{k}}$ is in the $z$-direction and the polarization of interest is in the $\mathrm{x}-\mathrm{y}$-plane.

electron density of the ionosphere is often perturbed by enhancements and depletions ranging in scale sizes from several meters to several hundred kilometers. Various modelling efforts have been undertaken to determine the effect of these structures on the received signal. For example, Wang et al. (2003) studied the signature of ionospheric structures on the received power, signal delay, and direction of arrival. Thorough studies of data and modelling from a previous HF transionospheric experiment performed by the ISIS II satellite have also been performed by James (2006), James et al. (2006), Gillies (2006), and Gillies et al. (2007). In these studies, the experimentally measured Faraday rotation and differential signal delay of radio waves was compared to the same parameters as determined by computational models.

In the present paper, the polarization state of the SuperDARN radars is examined and more specifically the relative amount of transmitted power that will be distributed between each of the two magnetoionic modes of propagation is modelled. The relative power of each mode received by the RRI was thoroughly investigated for various transmitter frequencies and ionospheric electron density profiles. This knowledge of the relative power of the two propagation modes will greatly assist in interpretion of the RRI instrument observations. This research is also of interest for general SuperDARN science as it details the propagation environment. The Doppler velocity that SuperDARN detects is directly related to the index of refraction at the scattering location (Gillies et al., 2009). Since the two modes of propagation have different indices of refraction, knowledge of which mode is involved in the scattering process is of significance when interpreting line-of-sight velocity values derived from coherent $\mathrm{Su}$ perDARN scatter.

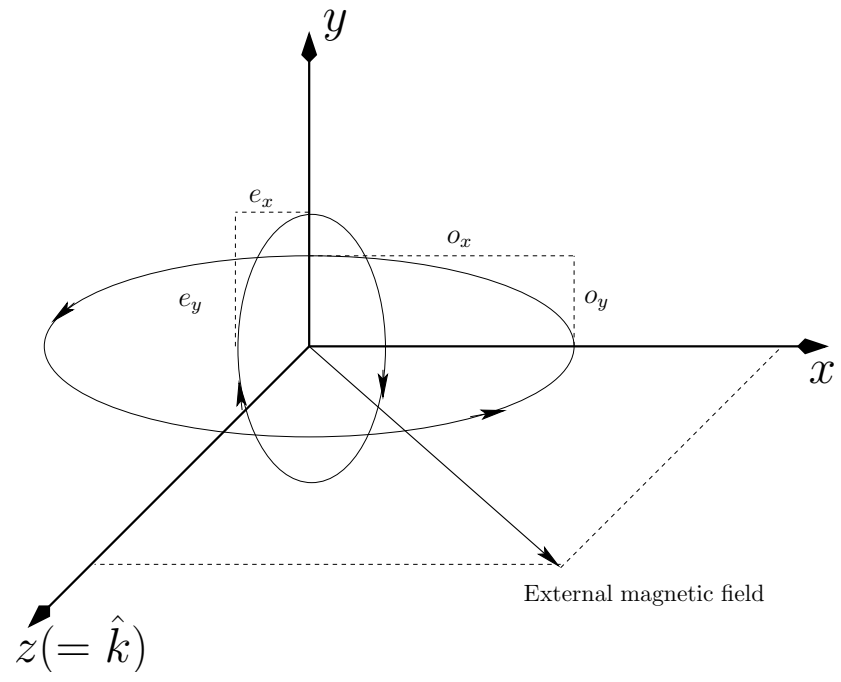

Fig. 2. The two magnetoionic modes predicted by Budden (1961) which exist from the decomposition of the wave in Fig. 1. The propagation direction of the modes $\hat{\boldsymbol{k}}$ is in the $z$-direction and the polarization of interest is in the $x-y$-plane.

\section{Determination of relative mode power}

This section has two objectives; namely, to define a general elliptical polarization state of a propagating magnetoionic wave (Fig. 1) at any point in the ionosphere using notation given by Budden (1961) and then to resolve this general polarization state into the two allowed magnetoionic propagation modes at that ionospheric location (Fig. 2). This decomposition will provide an expression for the amount of power that is injected into the $\mathrm{X}$-mode relative to the $\mathrm{O}$-mode, given an initial polarization state. This calculation is of use for the upcoming RRI experiment, as the RRI on ePOP will, at times, receive pulses from the two modes separately (the received pulses may overlap or be completely separate depending on factors such as: the pulse length, the transmitted frequency, and the ionospheric electron density).

The coordinate system used for this derivation is described by Budden (1961) and illustrated in Fig. 1. In this system, the $\mathrm{z}$-axis is oriented parallel to the wave propagation vector $(\hat{\boldsymbol{k}})$. The $\mathrm{x}$-axis is determined because the external magnetic field is taken to be in the $\mathrm{x}$-z-plane at an angle $\theta$ to the $\mathrm{z}$ axis. The $y$-axis follows from the definitions for the $\mathrm{x}$ - and $\mathrm{z}$ axes. In this geometry, Budden (1961) has shown that, when collisions are negligible, the two magnetoionic propagation modes are orthogonal elliptical polarization states in the $x-y$ plane and have principal axes along the $\hat{x}$ and $\hat{y}$ directions as demonstrated in Fig. 2.

A three-dimensional rotation is performed to transform the horizontally polarized SuperDARN wave in a local radar coordinate system into the Budden (1961) axes. This rotation allows the wave polarization state to be completely described 
in this axis system and determines the value for the orientation angle $\psi$ so that decomposition of the wave into the two allowed propagation modes can be performed.

The polarization ellipse of the wave in the principal axis frame has major and minor axes given by $a$ and $b$, respectively. In the principal axis frame (see Fig. 1), this initial polarization state (i.e., the polarization leaving the radar antennas) $\left(\vec{E}_{\mathrm{r}}\right)$ can be expressed as:

$\overrightarrow{E_{\mathrm{r}}}=\left(a e^{i \phi_{\mathrm{x}}} \hat{\mathrm{x}_{\mathrm{p}}}+b e^{i \phi_{\mathrm{y}}} \hat{\mathrm{y}_{\mathrm{p}}}\right) e^{i(\omega t-k z)}$

where $\phi_{\mathrm{x}}$ and $\phi_{\mathrm{y}}$ are the phases of the oscillation in the $\hat{\mathrm{x}_{\mathrm{p}}}$ and $\hat{y_{\mathrm{p}}}$ directions, respectively, and $\phi_{\mathrm{y}}-\phi_{\mathrm{x}}= \pm \pi / 2$, where the $( \pm)$ describes the sense of rotation of the polarization ellipse. Therefore, the polarization can be re-written as:

$\overrightarrow{E_{\mathrm{r}}}=\left(a \hat{\mathrm{x}_{\mathrm{p}}}+( \pm i) b \hat{\mathrm{y}_{\mathrm{p}}}\right) e^{i\left(\omega t-k z+\phi_{x}\right)}$.

A rotation of this ellipse must be performed to describe the polarization state in the Budden (1961) coordinate system which is defined using the propagation direction of the wave and the magnetic field orientation. Given a value for $\psi$ using coordinate transforms mentioned previously, the polarization can be described as:

$$
\begin{aligned}
\vec{E}_{\mathrm{r}}= & {[(a \cos \psi-( \pm i) b \sin \psi) \hat{x}} \\
& +(a \sin \psi+( \pm i) b \cos \psi) \hat{y}] e^{i\left(\omega t-k z+\phi_{x}\right)} .
\end{aligned}
$$

Equation (3) completely describes the polarization ellipse of Fig. 1.

The next step is to describe the polarization states of the two magnetoionic modes of propagation. This is accomplished using the wave-polarization ratio:

$\rho=\frac{\boldsymbol{E}_{\mathrm{y}}}{\boldsymbol{E}_{\mathrm{X}}}$

Where $\boldsymbol{E}_{\mathrm{x}}$ and $\boldsymbol{E}_{\mathrm{y}}$ are the phasor representations of the electric field along the $\mathrm{x}$ - and $\mathrm{y}$-axes, respectively. In the absence of collisions, $\rho$ is given by the following equation:

$\rho=i \frac{Y_{\mathrm{T}}^{2}}{2 Y_{\mathrm{L}}(1-X)} \pm i \sqrt{\frac{Y_{\mathrm{T}}^{4}}{4 Y_{\mathrm{L}}^{2}(1-X)^{2}}+1}$

or

$$
\rho=i \frac{(Y \sin \theta)^{2}}{2 Y \cos \theta(1-X)} \pm i \sqrt{\frac{(Y \sin \theta)^{4}}{4(Y \cos \theta)^{2}(1-X)^{2}}+1},
$$

where $X=\left(\omega_{p} / \omega\right)^{2}, Y=\omega_{c} / \omega, Y_{\mathrm{L}}=\left(\omega_{c} / \omega\right) \cos \theta$, and $Y_{\mathrm{T}}=\left(\omega_{c} / \omega\right) \sin \theta$ (note that $X$ represents the ratio of $\omega_{p}^{2}$ to $\omega^{2}$, not the X-mode of propagation). Further: $\omega_{\mathrm{c}}$ is the cyclotron frequency, $\omega_{\mathrm{p}}$ is the plasma frequency, $\omega$ is the radio wave frequency, and $\theta$ is the aspect angle. The major axis of the $\mathrm{O}$-mode is oriented along the $\mathrm{x}$-axis (i.e., the $\mathrm{O}$-mode major axis is always parallel to the component of the external $\mathrm{B}$-field that is perpendicular to $\hat{\boldsymbol{k}}$ ) and the major axis of the $\mathrm{X}$-mode is oriented along the $\mathrm{y}$-axis (this behaviour is illustrated in Fig. 2). This condition indicates that, with respect to Eq. (4), the absolute value of $\rho$ for the O-mode is always less than one and the absolute value of $\rho$ for the $\mathrm{X}$-mode is always greater than one. Both values for $\rho$ are purely imaginary, indicating that the electric field oscillations along the $\mathrm{x}$ - and $\mathrm{y}$-axes are $90^{\circ}$ out of phase for either mode.

When a radar wave is propagating nearly (or exactly) parallel or anti-parallel to the magnetic field lines (the aspect angle $\theta$ is near $0^{\circ}$ or $180^{\circ}$ ), the two polarization modes become circularly polarized waves with opposite sense. In the other extreme, when the radar wave propagates nearly (or exactly) perpendicular to the magnetic field, the polarization state of a mode is linear, each orthogonal to the other, assuming there are no collisions. In between these two propagation regimes, each mode takes on an elliptical polarization state which, if there are no collisions, is orthogonal to the other mode.

The polarizations of the two modes of propagation $\left(\overrightarrow{E_{\mathrm{O}}}\right.$ for the O-mode and $\vec{E}_{\mathrm{e}}$ for the X-mode) are described by:

$\overrightarrow{E_{\mathrm{o}}}=\left(o_{\mathrm{x}} e^{i \phi_{o_{\mathrm{x}}}} \hat{x}+o_{\mathrm{y}} e^{i \phi_{o_{\mathrm{y}}}} \hat{y}\right) e^{i(\omega t-k z)}$

and

$\overrightarrow{E_{\mathrm{e}}}=\left(e_{\mathrm{x}} e^{i \phi_{e_{\mathrm{x}}}} \hat{x}+e_{\mathrm{y}} e^{i \phi_{e_{\mathrm{y}}}} \hat{y}\right) e^{i(\omega t-k z)}$

$o_{\mathrm{x}}$ and $o_{\mathrm{y}}$ represent the major and minor axes of the O-mode ellipse and similarly, $e_{\mathrm{x}}$ and $e_{\mathrm{y}}$ represent the major and minor axes of the X-mode ellipse (note that $e_{\mathrm{x}}$ and $e_{\mathrm{y}}$ represent the axes of the X-mode ellipse should not be confused with the base of the natural $\log$ arithm $e$ ). Equation (4) gives the relation between the phasor representations of the oscillations along the $\mathrm{x}$ - and $\mathrm{y}$-axes for each mode:

$\rho_{\mathrm{oc}}=\frac{o_{\mathrm{y}} e^{i \phi_{o_{\mathrm{y}}}}}{o_{\mathrm{x}} e^{i \phi_{o_{\mathrm{x}}}}}$

and

$\rho_{\mathrm{ec}}=\frac{e_{\mathrm{y}} e^{i \phi_{e_{\mathrm{y}}}}}{e_{\mathrm{x}} e^{i \phi_{e_{\mathrm{x}}}}}$

where $\rho_{\mathrm{oc}}$ and $\rho_{\mathrm{ec}}$ are the axis ratios for the $\mathrm{O}$ - and X-modes from Eq. (6), respectively. Substitution gives,

$\overrightarrow{E_{\mathrm{O}}}=o_{\mathrm{X}} e^{i \phi_{o_{\mathrm{X}}}}\left(\hat{x}+\rho_{\mathrm{oc}} \hat{y}\right) e^{i(\omega t-k z)}$

and

$\overrightarrow{E_{\mathrm{e}}}=e_{\mathrm{X}} e^{i \phi_{e_{\mathrm{X}}}}\left(\hat{x}+\rho_{\mathrm{ec}} \hat{y}\right) e^{i(\omega t-k z)}$.

Recall that, because collisions have been neglected, $\rho_{\mathrm{oc}}$ and $\rho_{\mathrm{ec}}$ are imaginary numbers and have opposite signs to represent the opposite sense of rotation of the two modes. For simplicity, the imaginary portion of the two wave-polarization ratios should be explicitly written, so two new variables will be defined as $i \rho_{\mathrm{o}}=\rho_{\mathrm{oc}}$ and $i \rho_{\mathrm{e}}=\rho_{\mathrm{ec}}$ where the values for $\rho_{\mathrm{o}}$ and $\rho_{\mathrm{e}}$ are purely real. Also, for simplicity, the phases $\phi_{o_{\mathrm{x}}}$ and $\phi_{e_{\mathrm{x}}}$ will be written as $\phi_{\mathrm{o}}$ and $\phi_{\mathrm{e}}$. The polarizations of the two modes become:

$\vec{E}_{\mathrm{o}}=o_{\mathrm{x}} e^{i \phi_{\mathrm{o}}}\left(\hat{x}+i \rho_{\mathrm{o}} \hat{y}\right) e^{i(\omega t-k z)}$ 
and

$\overrightarrow{E_{\mathrm{e}}}=e_{\mathrm{x}} e^{i \phi_{\mathrm{e}}}\left(\hat{x}+i \rho_{\mathrm{e}} \hat{y}\right) e^{i(\omega t-k z)}$

The sense of rotation of the modes is opposite, indicating that $\rho_{\mathrm{o}}$ and $\rho_{\mathrm{e}}$ must have opposite signs, which Eq. (6) assures.

Now the initial polarization (Eq. 3) can be equated to the polarizations of the two modes of propagation:

$\overrightarrow{E_{\mathrm{r}}}=\overrightarrow{E_{\mathrm{o}}}+\overrightarrow{E_{\mathrm{e}}}$

Equating the real and imaginary components of both the $\mathrm{x}$ and $y$-axis values in Eq. (15) gives:

$a \cos \psi=o_{\mathrm{X}} \cos \phi_{\mathrm{O}}+e_{\mathrm{X}} \cos \phi_{\mathrm{e}}$,

$-( \pm) b \sin \psi=o_{\mathrm{X}} \sin \phi_{\mathrm{O}}+e_{\mathrm{X}} \sin \phi_{\mathrm{e}}$

$a \sin \psi=-\rho_{o} o_{\mathrm{X}} \sin \phi_{\mathrm{o}}-\rho_{e} e_{\mathrm{X}} \sin \phi_{\mathrm{e}}$,

$( \pm) b \cos \psi=\rho_{\mathrm{o}} o_{\mathrm{x}} \cos \phi_{\mathrm{o}}+\rho_{\mathrm{e}} e_{\mathrm{x}} \cos \phi_{\mathrm{e}}$

The knowns in these equations are $a, b,( \pm)$, and $\psi$, which define the initial conditions for the wave, (for example, for the initial SuperDARN polarization, $a=1.0, b=0.0$, the $( \pm)$ does not matter, and $\psi$ is calculated through the coordinate transform mentioned previously). Also known from the local magnetoionic conditions are $\rho_{\mathrm{o}}$ and $\rho_{\mathrm{e}}$ (from Eq. 6). The unknowns, which need to be solved to determine the relative power delivered to $\mathrm{X}$-mode, are $o_{\mathrm{X}}, e_{\mathrm{X}}, \phi_{\mathrm{O}}$, and $\phi_{\mathrm{e}}$. The solutions to these equations are:

$\phi_{\mathrm{o}}=\arctan \left[\tan \psi\left(\frac{( \pm) b \rho_{\mathrm{e}}-a}{( \pm) b-a \rho_{\mathrm{e}}}\right)\right]$

$o_{\mathrm{x}}=\frac{\sin \psi\left(( \pm) b \rho_{\mathrm{e}}-a\right)}{\sin \phi_{\mathrm{o}}\left(\rho_{\mathrm{o}}-\rho_{\mathrm{e}}\right)}$

$\phi_{\mathrm{e}}=\arctan \left[\tan \psi\left(\frac{( \pm) b \rho_{\mathrm{o}}-a}{( \pm) b-a \rho_{\mathrm{o}}}\right)\right]$

$e_{\mathrm{x}}=\frac{\sin \psi\left(( \pm) b \rho_{\mathrm{o}}-a\right)}{\sin \phi_{\mathrm{e}}\left(\rho_{\mathrm{e}}-\rho_{\mathrm{o}}\right)}$.

The values for $o_{\mathrm{y}}$ and $e_{\mathrm{y}}$ are found using:

$o_{\mathrm{y}}=\left|\rho_{\mathrm{o}}\right| o_{\mathrm{x}}$

$e_{\mathrm{y}}=\left|\rho_{\mathrm{e}}\right| e_{\mathrm{x}}$

Finally, the relative power that is delivered to the $\mathrm{X}$-mode compared to the O-mode $\left(R_{\mathrm{e}}\right)$, which is the quantity that is discussed in the following sections, in units of $\mathrm{dB}$ is:

$R_{\mathrm{e}}=10 \log _{10}\left[\frac{e_{\mathrm{x}}^{2}+e_{\mathrm{y}}^{2}}{o_{\mathrm{x}}^{2}+o_{\mathrm{y}}^{2}}\right]$

\section{The SuperDARN radar system}

High-latitude ionospheric convection is continuously monitored by the Super Dual Auroral Radar Network (SuperDARN) set of radars. A SuperDARN radar consists of sixteen transmitting antennas which produce, at any given time, a single beam of dwell time $7 \mathrm{~s}$ (normal scan) or $3 \mathrm{~s}$ (fast scan). By means of a phasing matrix, the beam is progressively shifted by $3.25^{\circ}$ through 16 successive beam positions, thereby covering an azimuth range of $52^{\circ}$ during a single complete scan. The frequency range of the radars is 8-20 MHz (Greenwald et al., 1995).

The antenna beam pattern of a SuperDARN array is designed for poleward viewing at elevation angles less than $\sim 45^{\circ}$. In practice, power is radiated from the horizontal antennas through all elevation angles from $0-180^{\circ}$ (e.g., Arnold et al., 2003). This is beneficial for the ePOP mission as the RRI can therefore receive transmitted signal throughout a pass over a given SuperDARN radar. The electric field polarization of the SuperDARN antennas is linear and oriented horizontal to the Earth. Since the geomagnetic field lines are nearly vertical at higher latitudes, the SuperDARN transmitted linear horizontal polarization is roughly perpendicular to the magnetic field lines. According to the magnetoionic theory, for propagation perpendicular to the magnetic field lines, the horizontal waves transmitted northward are predominantly extraordinary mode (X-mode) signals before entering the ionosphere.

\subsection{SuperDARN mode power distribution}

The equations for relative mode power derived in Sect. 2 were used to determine the power that a SuperDARN radar distributes to either mode of propagation. Inspection of Eq. (6) indicates that the polarization state with which the two modes propagate is dependent on electron density $N_{\mathrm{e}}$ $\left(\omega_{\mathrm{p}}=\sqrt{e^{2} N_{\mathrm{e}} / \epsilon_{\mathrm{o}} m}\right)$, radar frequency $\omega$, and propagation direction $\theta$. As the radars are ground-based, the initial electron density and collision frequency were taken as zero and the polarization ratios of the modes depended only on $\theta$ and $\omega$. Equations (20) through (26), along with the polarization ratio (Eq. 6), were used to determine the relative mode power. The power distribution for the Saskatoon SuperDARN (geographic coordinates: $52.16^{\circ} \mathrm{N}, 106.53^{\circ} \mathrm{E}$, magnetic coordinates: $61.34^{\circ} \mathrm{N}, 45.26^{\circ} \mathrm{W}$ ) at various frequencies has been modelled. Figure 3 illustrates the relative X-mode power $\left(10 \log _{10}\left[\frac{e_{\mathrm{x}}^{2}+e_{\mathrm{y}}^{2}}{o_{\mathrm{x}}^{2}+o_{\mathrm{y}}^{2}}\right]\right)$ for $12.5 \mathrm{MHz}$ propagation at different elevation and azimuth angles. The directions of geographic north, west, south, and east are represented by "N", "W", "S", and "E", respectively. The edge of the large circle represents $0^{\circ}$ elevation angle, while the centre of the circle represents $90^{\circ}$ elevation angle or directly vertical. The solid and dashed arrows illustrate the direction to magnetic north and the boresight of the radar, respectively. An X-mode relative 


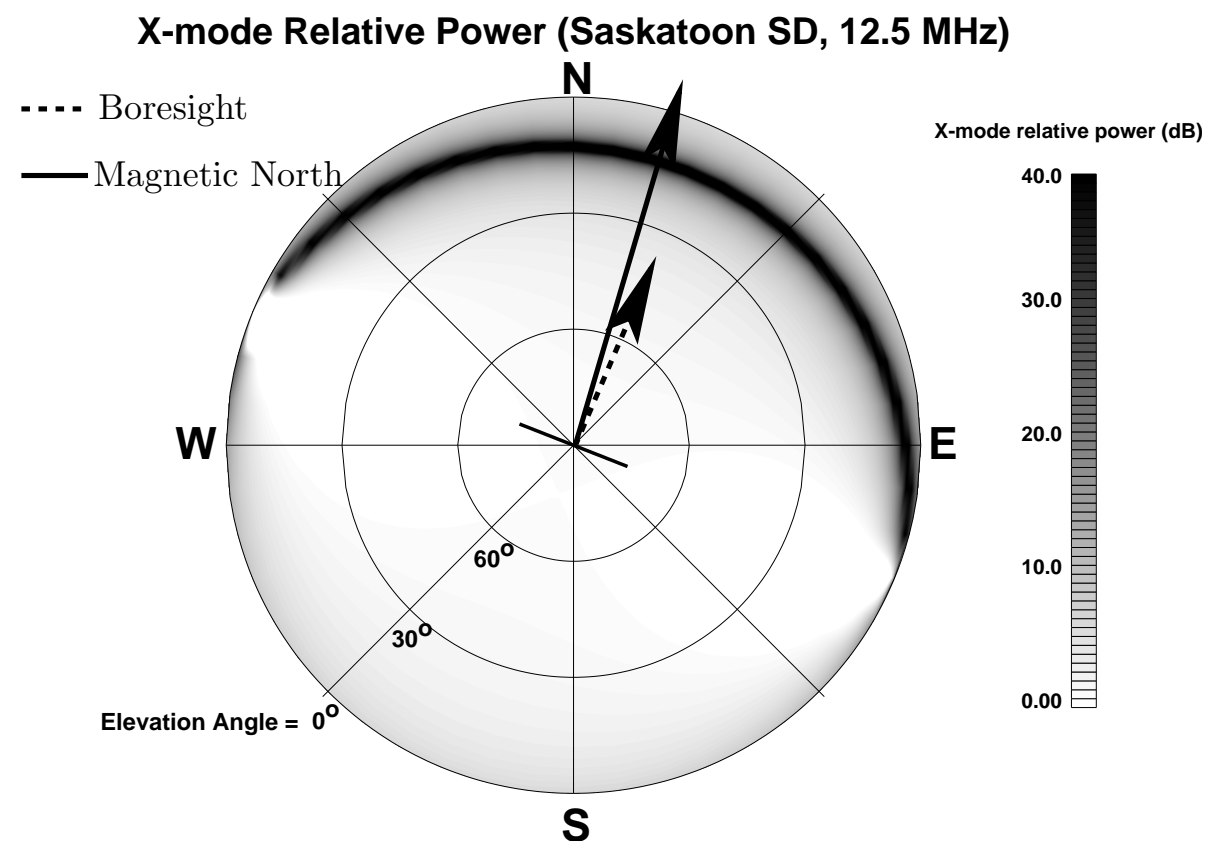

Fig. 3. Relative power delivered to the X-mode (Eq. 26) at $12.5 \mathrm{MHz}$ from the Saskatoon SuperDARN as a function of elevation and azimuth angles.

Table 1. Locations of Canadian SuperDARN radars.

\begin{tabular}{lrrrr}
\hline \multicolumn{1}{c}{ Radar } & $\begin{array}{r}\text { Latitude }\left({ }^{\circ} \mathrm{N}\right) \\
(\text { Geographic })\end{array}$ & $\begin{array}{r}\text { Longitude }\left({ }^{\circ} \mathrm{W}\right) \\
(\text { Geographic })\end{array}$ & $\begin{array}{r}\text { Latitude }\left({ }^{\circ} \mathrm{N}\right) \\
(\text { Geomagnetic })\end{array}$ & $\begin{array}{r}\text { Longitude }\left({ }^{\circ} \mathrm{W}\right) \\
(\text { Geomagnetic })\end{array}$ \\
\hline Saskatoon & 52.16 & 106.53 & 61.34 & 45.26 \\
Kapuskasing & 49.39 & 82.32 & 60.06 & 9.22 \\
Prince George & 53.98 & 122.59 & 59.88 & 65.67 \\
Rankin Inlet & 62.82 & 92.11 & 72.96 & 28.17 \\
Inuvik & 61.41 & 133.80 & 71.15 & 86.5 \\
\hline
\end{tabular}

power of $0.0 \mathrm{~dB}$ (white on the contour plot) would correspond to equal splitting between the two propagation modes. The elevation and azimuth angles which show darker shades on the plot indicate higher relative X-mode power is transmitted in these directions by SuperDARN.

From this figure, it is immediately apparent that there is a band of elevation angles north of the transmitter where the transmission is dominated by the X-mode. Meanwhile, above and south of the radar, the two transmission modes are roughly equal in power. It should also be noted that, near $0^{\circ}$ elevation angle and at azimuths that are nearly perpendicular to the boresight, there are two small regions in the plot in which the signal is dominated by the O-mode. This is due to the special geometry that exists in this location between the external magentic field, the propagation vector, and the initial wave polarization. However, since very little signal is transmitted in the direction parallel to the antenna, this region is of little importance. Modelling has also been performed for the other SuperDARN radars in Canada which are listed in Table 1. In general the results are quite similar to the Saskatoon radar modelling. The $\mathrm{X}$-mode relative power maximum is roughly centred on geomagnetic north and decreases in elevation for an increase in geomagnetic latitude. This behaviour is demonstrated in Figs. 4 and 5 which illustrate the relative $\mathrm{X}$-mode power at $12.5 \mathrm{MHz}$ for the Prince George (which is at a different longitude than the Saskatoon radar) and Rankin Inlet (which is at a high latitude) radars, respectively. It is clearly evident for the Prince George radar that the X-mode maximum power band is oriented to centre on magnetic north. Meanwhile the Rankin Inlet radar, which is the closest to the geomagnetic pole, shows an X-mode maximum band very close to the horizon.

Also noted (though not shown) was the effect of frequency on the mode power distributions. Transmitter frequencies of 


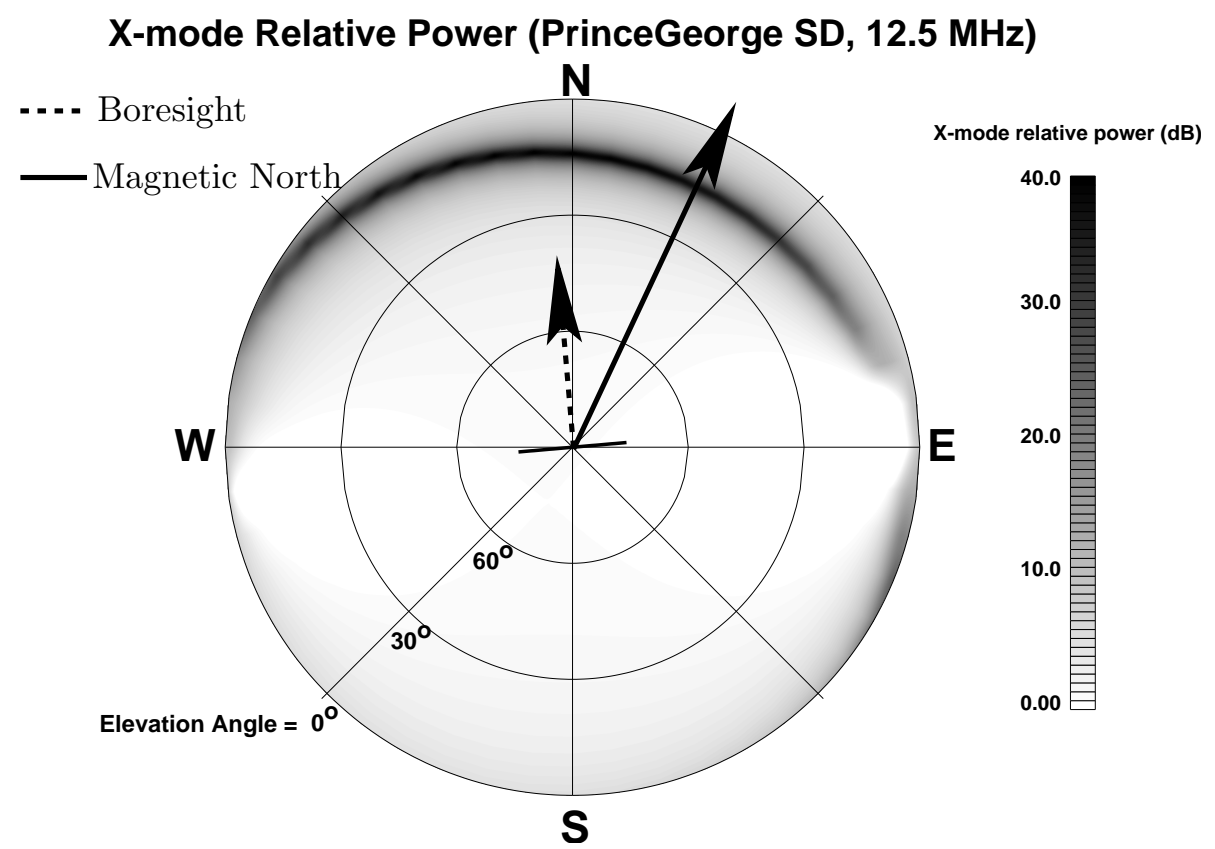

Fig. 4. Relative power delivered to the $\mathrm{X}$-mode (Eq. 26) at $12.5 \mathrm{MHz}$ from the Prince George SuperDARN as a function of elevation and azimuth angles.

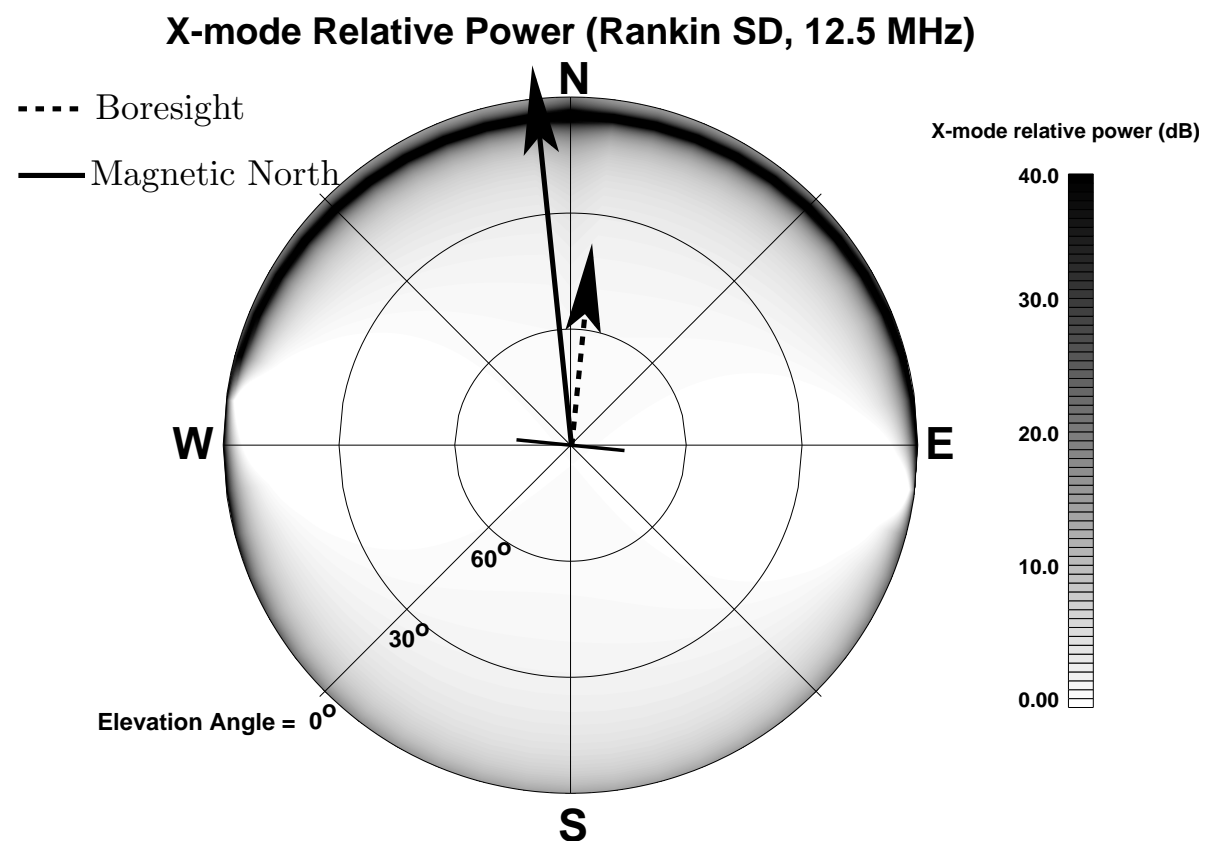

Fig. 5. Relative power delivered to the X-mode (Eq. 26) at $12.5 \mathrm{MHz}$ from the Rankin Inlet SuperDARN as a function of elevation and azimuth angles.

9.5 $\mathrm{MHz}, 12.5 \mathrm{MHz}$, and $14.5 \mathrm{MHz}$ were used in the mode power simulations. The main effect of frequency upon the distributions was a slight narrowing of the X-mode maximum band at higher frequencies, which will be discussed in more detail in Sect. 5.
It should be stressed that the power distributions in Figs. 3 through 5 illustrate only the relative power between the two modes and not the absolute transmitted power. The SuperDARN radars have complex antenna patterns and the absolute power of a transmitted signal will vary with launch 


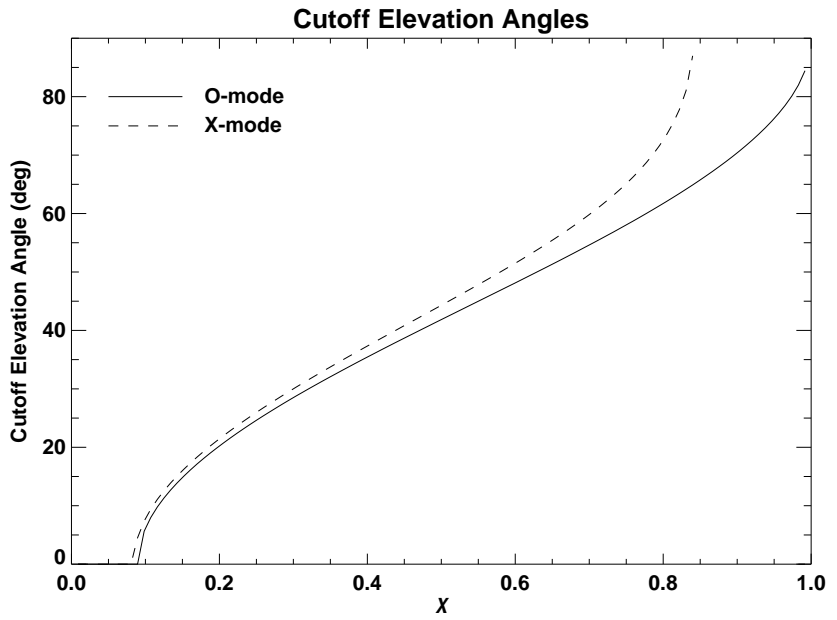

Fig. 6. Cutoff elevation angles as a function of the ratio $X$ (= $\left.\omega_{\mathrm{p}}^{2} / \omega^{2}\right)$ at the location of the peak electron density for the O-mode (lower curve) and X-mode (upper curve). Waves launched from the Saskatoon SuperDARN at lower elevation angles than these will be refracted back to Earth and not detected by the RRI on ePOP.

elevation and azimuth angles; however, the relative power between the modes as presented in Figs. 3 to 5 is still correct.

\section{Power distributions to be detected by RRI}

The preceeding section dealt with the power distributions of the $\mathrm{O}$ - and X-modes from a SuperDARN perspective. In order to focus this discussion on the ePOP/RRI experiment, the behaviour of the transmitted waves from SuperDARN as they propagate through the ionosphere to the orbiting RRI receiver was examined.

\subsection{Cutoff elevation angles}

The main finding of the modelling of the relative power, for either mode received by the RRI, was that waves launched at lower elevation angles will not escape the ionosphere. These waves will be internally reflected by the highly dense Fregion peak and thus will not be detected by the orbiting RRI receiver. The peak ionospheric electron density at SuperDARN latitudes can range from below $10^{11} \mathrm{~m}^{-3}$ to above $10^{12} \mathrm{~m}^{-3}$ (e.g., Hunsucker and Hargreaves, 2003). With this range of possible densities, the cutoff elevation angle (the minimum elevation angle for which a wave of a given frequency can propagate through the F-peak and reach the satellite) can vary substantially.

For a horizontally stratified ionosphere, the cutoff elevation angle may be found using Snell's Law in spherical coordinates (Bouguer's Law) (Born and Wolf, 1959):

$n_{\mathrm{o}} R_{\mathrm{o}} \cos \phi_{\mathrm{o}}=n_{\mathrm{p}} R_{\mathrm{p}} \cos \phi_{\mathrm{p}}$ where $n_{\mathrm{o}}, R_{\mathrm{o}}$, and $\phi_{\mathrm{o}}$ are the refractive index, radius (from the centre of the Earth), and elevation angle of the wave at the antenna location, respectively, and $n_{\mathrm{p}}, R_{\mathrm{p}}, \phi_{\mathrm{p}}$ have similar meanings at the location of the peak electron density in the ionosphere. For the SuperDARN radar, the initial index of refraction $n_{\mathrm{O}}$ on the ground can be taken as unity, and $R_{\mathrm{O}}$ is the radius of the Earth $R_{\mathrm{E}}$. When the signal at the cutoff elevation angle is totally internally reflected its propagation direction will be horizontal, indicating that $\phi_{\mathrm{p}}$ is $90^{\circ}$. Radar waves at higher elevation angles will escape the ionosphere and reach the satellite. The peak electron density occurs at roughly $h_{\text {scat }}=300 \mathrm{~km}$ so $R_{\mathrm{p}}=R_{\mathrm{E}}+h_{\text {scat }}$. The index of refraction in the ionosphere as given by the Appleton-Hartree equation (Budden, 1961) is

$$
n^{2}=1-\frac{X}{1-\frac{Y_{\mathrm{T}}^{2}}{2(1-X)} \pm \sqrt{\frac{Y_{\mathrm{T}}^{4}}{4(1-X)^{2}}+Y_{\mathrm{L}}^{2}}} .
$$

The variables $X, Y_{\mathrm{T}}$, and $Y_{\mathrm{L}}$ are as defined in Sect. 2. The positive sign in Eq. (28) corresponds to the O-mode of propagation and the negative sign to the $\mathrm{X}$-mode of propagation. In Fig. 6, Eqs. (27) and (28) are used to plot the cutoff elevation angle $\phi_{\mathrm{o}}$ for both modes of propagation versus the ratio $X\left(=\omega_{\mathrm{p}}^{2} / \omega^{2}\right)$, given a constant magnetic field strength of $5 \times 10^{-5} \mathrm{~T}$ and an aspect angle $\theta=80^{\circ}$. These values are representative of the Saskatoon SuperDARN radar wave propagation conditions. The top curve represents the X-mode cutoff elevation angle and the bottom curve represents the O-mode cutoff elevation angle. Only waves launched at elevation angles higher than those plotted in the figure for a given value of $X$ will escape the ionosphere and reach the RRI on ePOP. As such, the X-mode-only band near $12^{\circ}$ elevation angle that is evident in Fig. 3 will only be observable by the RRI when the value for the ratio $X$ at the location of peak F-region electron density is lower than $\sim 0.1$ (the figure indicates that waves launched at elevation angles higher than $\sim 10^{\circ}$ will escape the ionosphere). It should be noted that this cutoff angle calculation has assumed a horizontally stratified ionosphere. In reality, the F-region peak density will vary with latitude north and south of the radar.

Further affecting the transmitted signal as it propagates through the ionosphere is relative absorption of the modes in the lower ionosphere. Depending on the local time and the state of the ionosphere, this absorption can range from essentially zero to complete absorption. For quiet conditions $\left(K_{\mathrm{p}}<2\right.$ or 3 ), it is expected that the amount of absorption for either mode at SuperDARN frequencies will be less than $1 \mathrm{~dB}$. For slightly disturbed conditions $\left(3<K_{\mathrm{p}}<5\right)$, the absorption will be no more than $\sim 2 \mathrm{~dB}$ for the O-mode and $\sim 4 \mathrm{~dB}$ for the X-mode (James et al., 2006; Gillies et al., 2007). Finally, for very disturbed conditions $\left(K_{\mathrm{p}}>5\right.$ or 6$)$, both modes will be almost completely absorbed in the D- and E-regions and no signal will reach the RRI receiver. 
a)

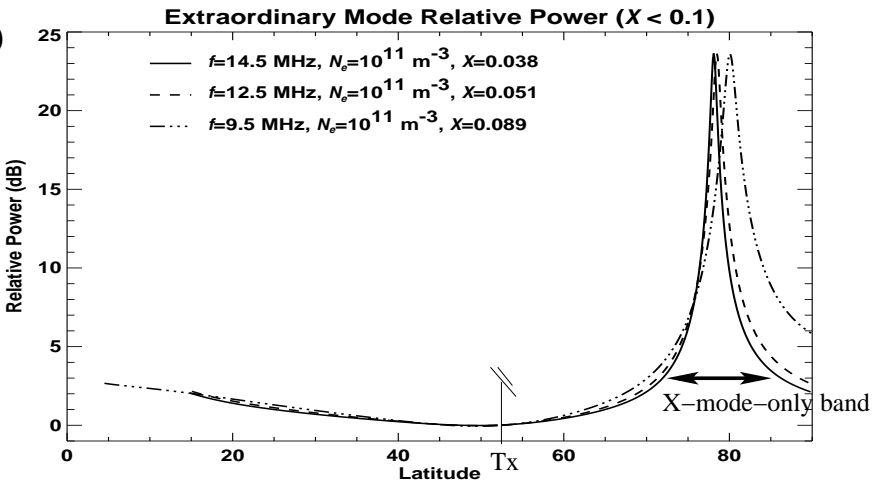

b) Extraordinary Mode Relative Power $(0.1<X<0.3)$

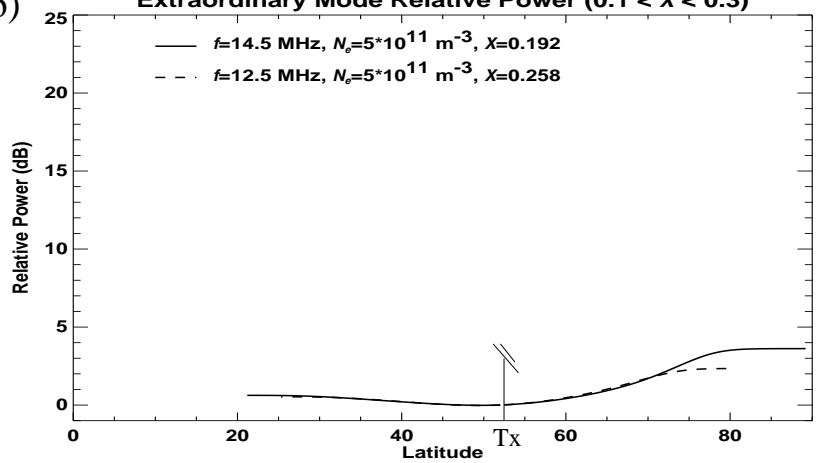

c)

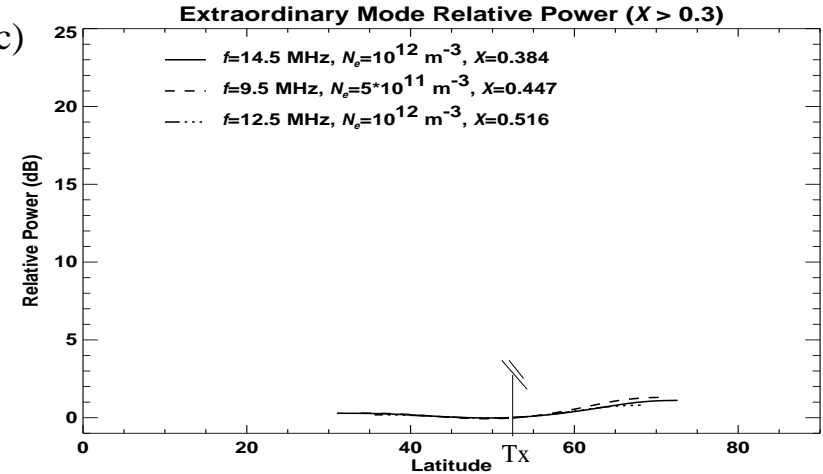

Fig. 7. Modelled X-mode relative power that will be received by the RRI on ePOP. Panels (a), (b), and (c) illustrate transmitter frequency and ionospheric peak density combinations which correspond to; $X<0.1,0.1<X<0.3$, and $0.3<X$, respectively. The modelling assumes a north-south satellite orbit at $1500 \mathrm{~km}$ directly over the Saskatoon SuperDARN radar (latitude $-52.16^{\circ} \mathrm{N}$ ).

\subsection{Raypath modelling of signal received by RRI}

The relative mode power that is to be received by the RRI on ePOP as it orbits over the Saskatoon SuperDARN radar has been modelled. A ray tracing program based on the ray equations from Haselgrove (1963) iteratively determined the path a wave would take from the transmitter on the ground to a given location of the satellite orbit. The program took as input the frequency to be transmitted, the starting (antenna) location, and an electron density profile. The electron density profiles for this study were determined from the International Reference Ionosphere (IRI) model (Bilitza, 2001). The magnetic field data for the ray trace program were obtained from the International Geomagnetic Reference Field (IGRF) model (Maus et al., 2005). For this study, the electron density distributions were horizontally stratified so they varied in altitude only. The iteration process was used to study a total of eight passes of the satellite over the transmitter at an orbital altitude of $1500 \mathrm{~km}$, the approximate apogee of the ePOP satellite. The $N_{\mathrm{e}}$ profile and transmitter frequency were varied for each pass. For both the $\mathrm{O}$ - and X-modes, rays which propagated from the SuperDARN radar location to the orbiting RRI receiver were calculated for $0.1^{\circ}$ latitude increments. Once these connecting rays were determined, the equations developed in Sect. 2 were used to model the relative mode power the RRI would observe in a pass over the transmitter.

Figure 7 illustrates the modelled relative power of the Xmode to that of the O-mode that would be detected by the RRI as a function of satellite latitude as it passes over the Saskatoon SuperDARN for a variety of electron density and frequency combinations. Absorption in the D- and E-regions has been taken into account; however, the electron density profiles were obtained for quiet conditions, so there was very little effect. It should be noted that Fig. 7 predicts equal splitting of power between the $\mathrm{O}$ - and $\mathrm{X}$-modes for the majority of the ePOP orbit, however when ionospheric conditions are appropriate for preferential absorption of the X-mode, the Omode signal that will be detected by the RRI will be higher than that of the X-mode in these locations. A further effect, which has not been considered, is focusing and defocusing of the waves as they propagate through the ionosphere (Hunsucker, 1991). It is possible that skip-distance focusing could preferentially favour the O-mode over the $\mathrm{X}$-mode, which may result in the $\mathrm{O}$-mode being received with a higher power than the X-mode in some instances. Future studies considering the amount of absorption for different levels of ionospheric activity and the effect of focusing and defocusing of the waves are being planned. 
In Fig. 7, different transmitter frequency and peak electron density combinations were modelled and are presented by the different curves in the three panels. The panels (a), (b), and (c) in the figure correspond to frequency-peak density combinations which result in; $X<0.1,0.1<X<0.3$, and $X>0.3$, respectively. The peak ionospheric electron density used to produce the values for $X$ in panel (a) was $10^{11} \mathrm{~m}^{-3}$ which is a reasonable value to expect in the nighttime ionosphere at SuperDARN observation latitudes. For these low values of $X(<0.1)$, the range of latitudes over which signal was detected was quite large and the X-mode-only band of signal was clearly observable. The curves in panel (b) in Fig. 7 were calculated using a peak electron density of $5 \times 10^{11} \mathrm{~m}^{-3}$, which is a reasonable estimate of peak $N_{\mathrm{e}}$ during evening or morning hours. At these moderate $X$ values $(0.1<X<0.3)$, the traced waves reached the satellite for a large range of latitudes, and the power was more evenly distributed between the $\mathrm{O}$ - and $\mathrm{X}$-modes. Finally, the curves in panel (c) were generated primarily using a profile with a peak $N_{\mathrm{e}}$ of $10^{12} \mathrm{~m}^{-3}$ which is representative of electron densities during the daytime. For the higher $X$ values $(>0.3)$, the range of latitudes over which the the signal was observed was much narrower. It should be noted that the given values of $X$ in Fig. 7 are not only dependent on local time as discussed, but also vary with time of year and location. Also, it was assumed in the generation of the profiles used in Fig. 7 that there was little auroral activity. Further, the SuperDARN transmitting frequency is chosen to provide the widest range of ionospheric scatter so that the more extreme values for $X$ may not often occur.

\section{Discussion}

\subsection{Relative mode power distribution of SuperDARN}

The plots of relative $\mathrm{X}$-mode power as a function of propagation direction in Figs. 3 to 5 illustrate the X-mode-only band. The middle of the band occurs in the direction where both propagation direction and the wave polarization electric field are perpendicular to the field lines. This behaviour is evident in Figs. 3, 4, and 5 which present the relative power delivered to the X-mode as a function of propagation direction for the Saskatoon, Rankin Inlet and Prince George radars, respectively. The reason that the X-mode-only band is centered on geomagnetic north relates to the geometry of the radar boresight with respect to the background magnetic field. Northward propagation of the waves places them nearly perpendicular to the field lines. Recall that in this regime, the two modes have orthogonal linear polarization states. The $\mathrm{O}$ mode polarization is oriented along the component of the magnetic field in the polarization plane, while the X-mode is oriented perpendicular to this component. Since the initial polarization of SuperDARN is linear and oriented horizontal to the surface of the Earth (or perpendicular to the nearly vertical magnetic field lines), it is basically an X-mode wave when leaving the antenna array. Therefore, it is not unexpected that the $\mathrm{X}$-mode would dominate the transmitted signal in this region. Conversely, above the transmitter and slightly southward, the wave propagates nearly along the magnetic field lines. In this regime, the two modes propagate as orthogonal circular polarization states. The summation of two circular waves of opposite sense will only produce the initial linear wave if they are of equal amplitude. Therefore, for propagation directions that are not perpendicular to the magnetic field lines, the two modes are of roughly equal power.

The effect of frequency on the mode power distributions was also noted (though not presented). An increase in transmitter frequency caused the X-mode-only band to narrow slightly. This was caused by the fact that, at higher frequencies, the band of aspect angles over which the modes take on nearly linear polarizations is much smaller.

It should also be noted that the index of refraction directly affects the Doppler velocity that is measured by SuperDARN (Gillies et al., 2009) and since the indices of refraction for the $\mathrm{O}$ - and X-modes are different, identifying which mode participates in coherent scatter is important. Since coherent scatter occurs due to field aligned irregularities, the SuperDARN wave must be perpendicular to the magnetic field lines to receive scatter. Therefore, scatter is received from elevation angles typically below $\sim 30^{\circ}$. Inspection of Figs. 3 through 5 indicates that it is primarily the X-mode which participates in ionospheric scatter. The refractive index of the X-mode is slightly lower than that of the O-mode, so it is important to use the correct value for refractive index when modifications are made to SuperDARN velocity estimates to account for this effect.

\subsection{Relative mode power detected by the RRI}

Figure 7 demonstrates the relative $\mathrm{X}$-mode power that is expected to be received by the RRI on ePOP as a function of latitude for various ionospheric conditions. For low values of $X(<0.1)$, the signal is expected to be observed over a large latitudinal range and there is a clear region of X-mode-only signal. This behaviour occurs because the computed waves are not bent substantially by the ionosphere and even low elevation angle waves reach the satellite (as could be predicted from Fig. 6). For slightly higher values of $X$, the signal is observed over a large range of latitudes, but the X-mode-only band is not apparent. In this range, the cutoff elevation angle is higher than the X-mode-only band observed in Fig. 3 so this band does not appear. Near the cutoff elevation angle, however, the traced waves are bent substantially by the ionosphere and this is the reason signal is predicted for a relatively large range of latitudes. When the values for $X$ are even higher $(>0.3)$ the signal is observed over a very short latitudinal range. In these cases, the cutoff elevation angles are quite large and the traced waves are not able to travel far 
north or south of the transmitter without being reflected back to Earth before reaching ePOP satellite altitudes.

It is apparent from Fig. 7 that the relative mode power received at RRI will be highly dependent on both frequency and electron density. As the transmitting frequency is known, an analysis of the relative mode power pattern as a function of latitude with reference to Fig. 7 will reveal the variations of the peak ionospheric electron density. If signal is received only over a narrow range of latitudes and the $\mathrm{O}$ - and X-modes are of equal power throughout the pass, a high $X$ value at the peak will be inferred. For example, a peak $X$ value of 0.3 or higher will correspond to a peak $N_{\mathrm{e}}$ value above $\sim 6 \times 10^{11} \mathrm{~m}^{-3}$ if the transmitter frequency is 12.5 MHz. However, if signal is received for a wide range of latitudes and there is a clear X-mode-only band present, then the peak $X$ value and thus the peak electron density will be low. For example, a peak $X$ value of less than 0.1 will only occur when the peak $N_{\mathrm{e}}$ value is below $\sim 2 \times 10^{11} \mathrm{~m}^{-3}$ for a transmitter frequency of $12.5 \mathrm{MHz}$.

Another benefit of the measurement of mode power will be the insight that it brings into the relative absorption of the two modes. For example, in the calculations for Fig. 7 it was assumed that there was a quiet ionosphere and thus little absorption of the signal in the D- and E-regions. However, if the X-mode is received with considerably less power than the O-mode at latitudes where the modelling indicated the modes should have roughly equal power, a measure of the amount of relative absorption that occurred can be obtained.

\section{Conclusions}

The polarization along the paths followed by SuperDARN rays has been studied in preparation for the upcoming ePOP satellite mission. The relative power of the two modes of propagation as a function of propagation direction and frequency has been calculated for each of the Canadian radars. It has been determined that the horizontal linearly polarized wave that is transmitted by the radars resembles the X-mode for propagation north of a transmitter. Therefore, there is a band of azimuths where the X-mode dominates the transmitted signal. This band of "X-mode-only" signal is oriented towards magnetic north and is at an elevation angle equal to the dip angle of the magnetic field lines at the radar. The width of this band narrows with increasing frequency. Meanwhile, above and south of the radars, the transmitted signal is split roughly equally between the $\mathrm{O}$ - and $\mathrm{X}$-modes. This is because the modes are circularly polarized states of roughly equal power resulting from the initial linear wave.

Ray tracing of a wave from the Saskatoon SuperDARN to the ePOP RRI receiver was performed for various frequencies and electron density profiles. The relative mode power that will be received as a function of satellite latitude was determined for various F-region peak $X\left(\omega_{\mathrm{p}}^{2} / \omega^{2}\right)$ values. It was found that, with an increase in $X$, the satellite observes signal over a narrower latitude range above the transmitter. Also, the $\mathrm{X}$-mode-only band is only apparent for low peak $X$ values. These calculations of relative mode power as a function of both satellite latitude and electron density will help characterize the ionospheric density profiles and provide a measure of relative absorption of the two propagation modes when the RRI receives signal from the SuperDARN ground transmitters.

It has also been found that for the northward propagation directions from SuperDARN which are most appropriate for ionospheric scatter, the X-mode dominates the transmitted signal. Since the index of refraction is an important factor for interpretation of the measured SuperDARN velocities (Gillies et al., 2009), the fact that the X-mode dominates the coherent scatter is an important result. Attempts to account for refractive index to improve the SuperDARN line-of-sight velocities must recognize that it is the refractive index for the $\mathrm{X}$-mode and not the $\mathrm{O}$-mode which must be applied to the measured velocities.

Acknowledgements. During this work RGG was supported by the Canadian Space Agency and the Natural Sciences and Engineering Research Council (NSERC) to the Canadian component of the SuperDARN ground based radar system and the CASSIOPE/ePOP project. International Reference Ionosphere (IRI) electron density profiles have been provided by the Space Physics Data Facility (SPDF).

Topical Editor K. Kauristie thanks P. Dyson and M. Uspensky for their help in evaluating this paper.

\section{References}

Arnold, N. F., Robinson, T. R., Lester, M., Byrne, P. B., and Chapman, P. J.: Super Dual Auroral Radar Network observations of fluctuations in the spectral distribution of near range meteor echoes in the upper mesosphere and lower thermosphere, Ann. Geophys., 19, 425-434, 2001,

http://www.ann-geophys.net/19/425/2001/.

Bilitza, D.: International Reference Ionosphere 2000, Radio Sci., 36, 261-275, 2001.

Budden, K. G.: Radio Waves in the Ionosphere, Cambridge University Press, 1961.

Gillies, R. G., Hussey, G. C., Sofko, G. J., McWilliams, K. A., Fiori, R. A. D., Ponomarenko, P., and St.-Maurice, J.-P.: Improvement of SuperDARN velocity measurements by estimating the index of refraction in the scattering region using interferometry, J. Geophys. Res., 114, A07305, doi:10.1029/2008JA013967, 2009.

Gillies, R. G., Hussey, G. C., James, H. G., Sofko, G. J., and André, D.: Modelling and observation of transionospheric propagation results from ISIS II in preparation for ePOP, Ann. Geophys., 25, 87-97, 2007, http://www.ann-geophys.net/25/87/2007/.

Gillies, R. G.: Modelling of Transionospheric HF Radio Wave Propagation for the ISIS II and ePOP Satellites, M.Sc. Thesis, Institute of Space and Atmospheric Studies, University of Saskatchewan, 2006.

Greenwald, R. A., Baker, K. B., Dudeney, J. R., Pinnock, M., Jones, T. B., Thomas, E. C., Villain, J.-P., Cerisier, J. C., Senior, C., 
Hanuise, C., Hunsucker, R. D., Sofko, G., Koehler, J., Nielsen, E., Pellinen, R., Walker, A. D. M., Sato, N., and Yamagishi, H.: DARN/SuperDARN: A Global View of the Dynamics of HighLatitude Convection, Space Sci. Rev., 71, 761-796, 1995.

Haselgrove, J.: The Hamiltonian ray path equations, J. Atmos. Terr. Phys., 25, 397-399, 1963.

Hunsucker, R. D.: Radio Techniques for Probing the Terrestrial Ionosphere, Springer-Verlag Berlin Heidelberg, 1991.

Hunsucker, R. D. and Hargreaves, J. K.: The High-Latitude Ionosphere and its Effects on Radio Propagation, Cambridge University Press, 2003.

James, H. G.: Effects on transionospheric HF propagation observed by ISIS at middle and auroral latitudes, Adv. Space Res., 38(11), 2303-2312, 2006.

James, H. G.: High-frequency direction finding in space, Review of Scientific Instruments, 74(7), 3478-3486, 2003.

James, H. G., Gillies, R. G., Hussey, G. C., and Prikryl, P.: HF fades caused by multiple wave fronts detected by a dipole antenna in the ionosphere, Radio Sci., 41, RS4018, doi:10.1029/2005RS003385, 2006.
James, H. G. and Lunscher, W. H. H. J.: A digital radio receiver for ionospheric research, NATO Specialist Meeting IST-056, "Characterizing the Ionosphere", Paper 23, Fairbanks, AK, USA, 1216 June 2006.

Maus, S., Macmillan, S., Chernova, T., Choi, S., Dater, D., Golovkov, V., Lesur, V., F. Lowes, Luhr, H., Mai, W., McLean, S., Olsen, N., Rother, M., Sabaka, T., Thomson, A., and Zvereva, T.: The 10th generation international geomagnetic reference field, Phys. Earth Planet. Int., 151, 320-322, 2005.

Wang, L., MacDougall, J. W., and James, H. G.: Ionospheric structure effects on HF radio wave propagation for the Enhanced Polar Outflow Probe (e-POP) satellite mission, Radio Sci., 39, RS2019, doi:10.1029/2003RS002975, 2004.

Yau, A. W, James, H. G., and Lui, W.: The Canadian Enhanced Polar Outflow Probe (ePOP) mission in ILWS, Adv. Space Res., 38(8), 1870-1877, 2006. 